\title{
On the departure process of a leaky bucket system with long-range dependent input traffic*
}

\author{
Socrates Vamvakos and Venkat Anantharam ** \\ EECS Department, University of California, Berkeley, CA 94720, USA \\ E-mail: sokratis@cory.eecs.berkeley.edu; ananth@vyasa.eecs.berkeley.edu
}

\begin{abstract}
Due to the strong experimental evidence that the traffic to be offered to future broadband networks will display long-range dependence, it is important to study the possible implications that such traffic may have for the design and performance of these networks. In particular, an important question is whether the offered traffic preserves its long-range dependent nature after passing through a policing mechanism at the interface of the network. One of the proposed solutions for flow control in the context of the emerging ATM standard is the so-called leaky bucket scheme. In this paper we consider a leaky bucket system with long-range dependent input traffic. We adopt the following popular model for long-range dependent traffic: Time is discrete. At each unit time a random number of sessions is initiated, having the distribution of a Poisson random variable with mean $\lambda$. Each of these sessions has a random duration $\tau$, where the integer random variable $\tau$ has finite mean, infinite variance, and a regularly varying tail, i.e., $P(\tau>k) \sim k^{-\alpha} L(k)$, where $1<\alpha<2$ and $L(\cdot)$ is a slowly varying function. Once a session is initiated, it generates one cell at each unit of time until its termination. We examine the departure process of the leaky bucket policing mechanism driven by such an arrival process, and show that it too is long-range dependent for any token buffer size and any - finite or infinite - cell buffer size. Moreover, upper and lower bounds for the covariance sequence of the output process are established. The above results demonstrate that long-range dependence cannot be removed by the kinds of flow control schemes that are currently being envisioned for broadband networks.
\end{abstract}

Keywords: ATM networks, flow control, leaky bucket scheme, long-range dependence, regularly varying tails

\section{Introduction - problem formulation}

Recent experimental studies of traffic to be carried by broadband networks have pointed out the importance of analyzing the performance of communication networks using traffic models with long-range dependence. Long-range dependence in network traffic has been reported, for instance, in [7], where statistical analysis of measurements of Ethernet traffic at Bellcore demonstrated its self-similar nature; in [3] long-range dependence has been established in variable bit rate video traffic generated by a number

${ }^{*}$ Research supported by NSF under NCR 9422513 and by a U.C. Berkeley Regents Fellowship.

** Address all correspondence to the second author.

(C) J.C. Baltzer AG, Science Publishers 


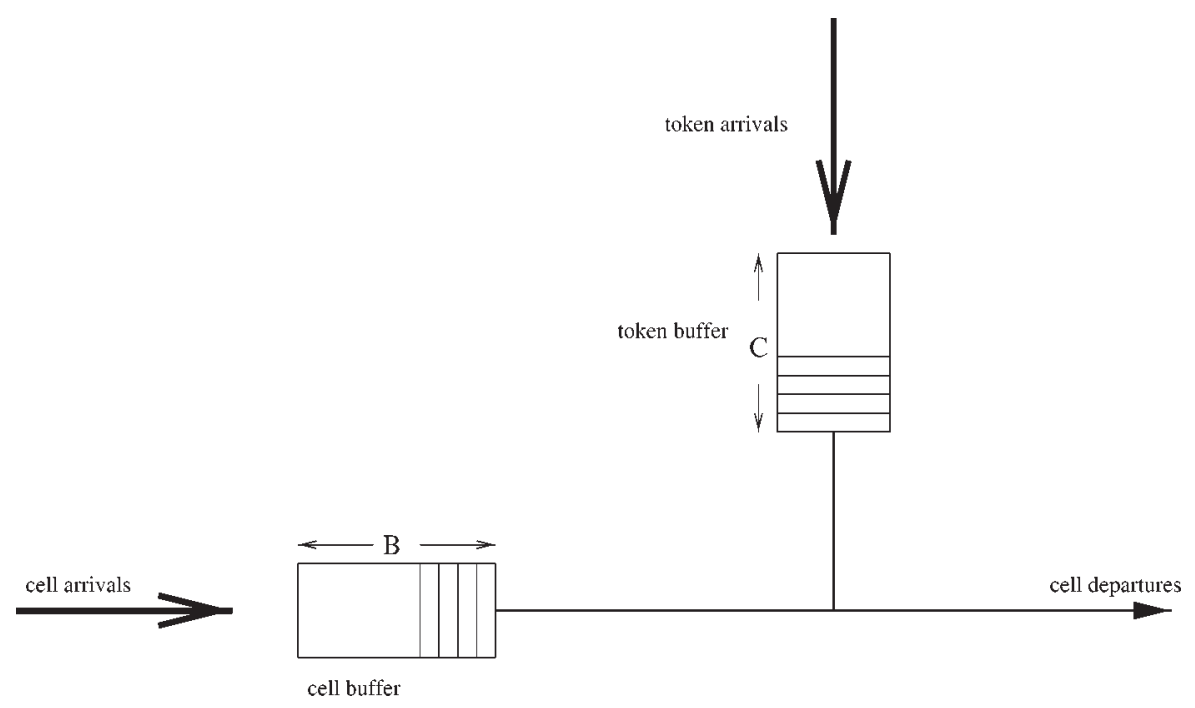

Figure 1. The leaky bucket scheme.

of different codecs; and in [11] the presence of long-range dependence in TELNET and other wide area network traffic was concluded.

In the light of this strong experimental evidence it is important to examine in more detail the possible implications that long-range dependent traffic may have on the design and performance of broadband networks. In particular, an important question is whether the offered traffic preserves its long-range dependent nature after passing through a policing mechanism at the interface of the network. One of the proposed solutions for flow control in the context of the emerging ATM standard is the so-called leaky bucket scheme which is shown schematically in figure 1. Cells of fixed size arrive at a buffer of size $B \geqslant 0$. The departure of cells from the buffer is controlled by tokens that arrive at a buffer of fixed size $C \geqslant 1$. An arriving cell can be transmitted only if it finds a token in the token buffer, in which case it is transmitted instantaneously by consuming a token. If the token buffer is empty, the cell has to wait for the generation of a new token. Time is assumed to be discrete and exactly one token is generated at the beginning of each unit of time. A stored cell is transmitted immediately upon the generation of a new token. Moreover, the outgoing capacity of the link is assumed to be at least $C$, so that it imposes no limitations on the number of cells that can be transmitted instantaneously.

We assume that the cell arrival process belongs to a class of discrete-time longrange dependent traffic models which includes as a special case the one proposed in [8]. In an arrival model of this class a random number of sessions is initiated at the beginning of each unit of time, distributed as a Poisson random variable with mean $\lambda$. Each of these sessions has a random duration $\tau$, where the integer random variable $\tau$ has finite mean, infinite variance, and a regularly varying tail, i.e., $P(\tau>k)$ $\sim k^{-\alpha} L(k)$, where $1<\alpha<2$ and $L(\cdot)$ is a slowly varying function. Once a session is 
(1) Immediately rejected if token buffer is full.

(2) Includes cells from sessions that start at time $\mathrm{k}$ and sessions that started prior to time $\mathrm{k}$.

(3) Departure variable $d$ is computed.

(4) Excess cells are discarded in the case of finite B.

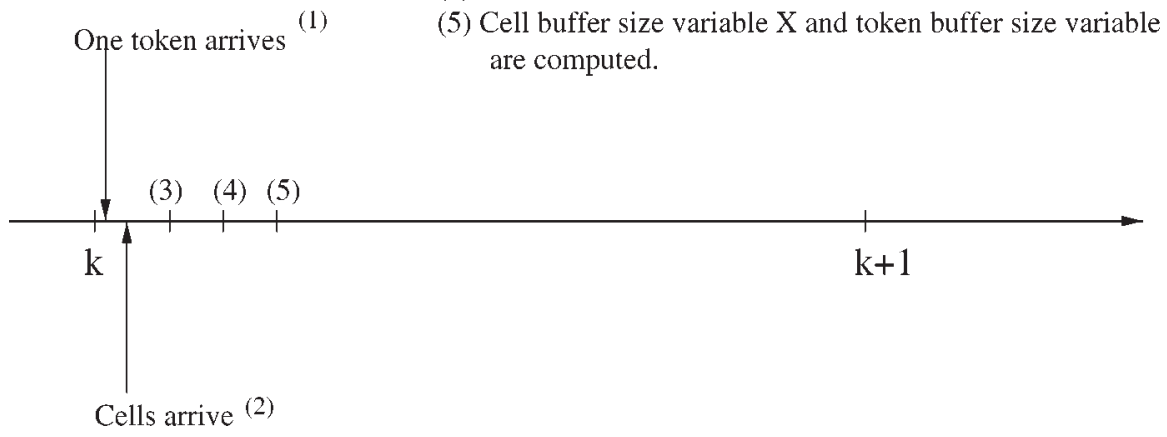

Figure 2. Timing conventions.

initiated, it generates one cell at the beginning of each unit of time until its termination. We will also consider the case of infinite cell buffer, $B=\infty$, in which case the stability condition $\lambda E \tau<1$ is assumed to hold. According to the results in [2,9], we may consider a stationary regime in which the departure process is stationary and ergodic - this will become clear once we write the system equations later in the paper.

Following Cox [5], a stationary second-order stochastic process is defined to be long-range dependent if the sum of the absolute values of its covariances is infinite, i.e.,

$$
\sum_{m=1}^{\infty}|r(m)|=\infty
$$

where $r(m)$ the covariance function of the process. In this paper we analyze a leaky bucket system with the arrival process as discussed above; a straightforward calculation shows that this arrival process is long-range dependent, see, e.g., Cox [5]. We examine the stationary departure process of the system and show that it too is long-range dependent.

Before going on to the statement of our results and the detailed proofs, we introduce the timing conventions and notation we will use, see figure 2 .

By convention, we assume that at the beginning of each slot a new token arrives, and that this is discarded immediately if the token buffer is full. The cells of the input process are assumed to arrive immediately after the token. The departure process is measured immediately after the maximum possible number of cells is served. After this, if there are more cells than can be stored in the cell buffer, these cells are discarded.

In what follows, we will denote by $d^{(C, B)}(k)$ the number of departures at time $k$ of a leaky bucket system with token buffer size $C$ and cell buffer size $B$. We let $X^{(C, B)}(k),\left(Y^{(C, B)}(k)\right)$ denote the number of cells (tokens) in the cell (token) buffer at 
time $k$ in such a system. These quantities are measured after the cell departures and the cell rejections at time $k$, see figure 1 .

In section 2 we consider the case of where the cell buffer is infinite, i.e., $B=\infty$. In section 3 we consider the case of finite cell buffer, i.e., $\infty>B \geqslant 0$. Throughout, we assume that $C \geqslant 1$. The explanation for this is that, with our timing conventions, in the case $C=0$ there would never be any tokens to serve cells.

Given two functions $f(m)$ and $g(m)$ we write $f(m) \sim g(m)$ for $\lim _{m \rightarrow \infty}(f(m) /$ $g(m))=1$ (we have already used this notation above). We write $f(m) \gtrsim g(m)$ for $\liminf _{m \rightarrow \infty}(f(m) / g(m)) \geqslant 1$ and $f(m) \lesssim g(m)$ for $\limsup _{m \rightarrow \infty}(f(m) / g(m)) \leqslant 1$.

\section{Infinite cell buffer case}

In this section we will consider the case $B=\infty$. We first examine the departure process in the case $C=1$. In this case the leaky bucket system is equivalent to a single-server queue that is served at a constant rate equal to one cell per unit time. Thus we have one of the cases analyzed in [1], (i.e., the case $s=1$ in the notation of [1]). We prove the following:

Lemma 1. The departure process of the leaky bucket system in the case $C=1$ is long-range dependent. Moreover, if we denote by $r^{(1, \infty)}(m)$ the covariance of the output process for lag $m$, then there are constants $K_{1}$ and $K_{2}$ such that

$$
r^{(1, \infty)}(m) \gtrsim K_{1} m^{1-\alpha} L(m)
$$

and

$$
r^{(1, \infty)}(m) \lesssim K_{2} m^{1-\alpha} L(m) .
$$

$K_{1}$ and $K_{2}$ can be explicitly estimated in terms of the parameters of the problem.

Proof. Consider the stationary departure process $\left\{d^{(1, \infty)}(k)\right\}$. Since $C=1$, we have $d^{(1, \infty)}(k) \in\{0,1\}, \forall k$. Moreover, the expected value of the departure process is equal to that of the input process in stationarity, since the stability condition holds, i.e.,

$$
E\left[d^{(1, \infty)}(k)\right]=\lambda E \tau .
$$

The covariance of the departure process is

$$
\begin{aligned}
r^{(1, \infty)}(m)= & E\left[\left(d^{(1, \infty)}(k)-\lambda E \tau\right)\left(d^{(1, \infty)}(k+m)-\lambda E \tau\right)\right] \\
= & (\lambda E \tau)^{2} P\left(d^{(1, \infty)}(k+m)=0, d^{(1, \infty)}(k)=0\right) \\
& -(\lambda E \tau)(1-\lambda E \tau) P\left(d^{(1, \infty)}(k+m)=0, d^{(1, \infty)}(k)=1\right) \\
& -(\lambda E \tau)(1-\lambda E \tau) P\left(d^{(1, \infty)}(k+m)=1, d^{(1, \infty)}(k)=0\right) \\
& +(1-\lambda E \tau)^{2} P\left(d^{(1, \infty)}(k+m)=1, d^{(1, \infty)}(k)=1\right) .
\end{aligned}
$$


If we define $P_{i \mid j}^{m} \triangleq P\left(d^{(1, \infty)}(k+m)=i \mid d^{(1, \infty)}(k)=j\right)$ and take into account that because of (4) we have $P\left(d^{(1, \infty)}(k)=0\right)=1-\lambda E \tau, P\left(d^{(1, \infty)}(k)=1\right)=\lambda E \tau$, we get from the above relation

$$
\begin{aligned}
r^{(1, \infty)}(m)= & (\lambda E \tau)^{2}(1-\lambda E \tau) P_{0 \mid 0}^{m}-(\lambda E \tau)^{2}(1-\lambda E \tau) P_{0 \mid 1}^{m} \\
& -(\lambda E \tau)(1-\lambda E \tau)^{2} P_{1 \mid 0}^{m}+(\lambda E \tau)(1-\lambda E \tau)^{2} P_{1 \mid 1}^{m} \\
= & (\lambda E \tau)(1-\lambda E \tau)\left(P_{0 \mid 0}^{m}-P_{0 \mid 1}^{m}\right) \\
= & (\lambda E \tau)(1-\lambda E \tau)\left(P_{1 \mid 1}^{m}-P_{1 \mid 0}^{m}\right) .
\end{aligned}
$$

In the above we used the fact that

$$
P_{0 \mid 0}^{m}+P_{1 \mid 0}^{m}=P_{0 \mid 1}^{m}+P_{1 \mid 1}^{m}=1
$$

From equation (6) one can see that $r^{(1, \infty)}(m)$ is positive, since $0<\lambda E \tau<1$ and $P_{1 \mid 1}^{m}>P_{1 \mid 0}^{m}$, which is proved in proposition 1 .

Consider now equation (6). First note that $d^{(1, \infty)}(k)=0$ means that the cell buffer at the time instant $k$ is empty. This is because sessions continuously bring in cells, so that the absence of a departure at time $k$ implies there are no active sessions among the sessions that arrived at or before time $k$, and therefore the system will never again have to handle cells corresponding to sessions that arrived at or before time $k$, so that in particular there are no cells left in the system at time $k$. We will henceforth refer to cells belonging to sessions that have been initiated at or before time $k$ as cells of type 1 , while cells belonging to sessions that arrived after time $k$ will be referred to as cells of type 2. Thus, if $d^{(1, \infty)}(k)=0$ there are no cells of type 1 in the system from time $k$ onwards. Assume that beginning at time $k+1$ priority is given to cells of type 2 over cells of type 1 ; this does not change the departure process, which only counts the total number of cells departing at any time. If at some point there are no cells of type 2 in the system, then the service of any remaining cells of type 1 will be resumed. Then we claim the following:

Proposition 1. For any $m \geqslant 1$ we have

$$
P_{1 \mid 1}^{m}-P_{1 \mid 0}^{m}=P\left\{A \text { cell of type } 1 \text { departs at time } k+m \mid d^{(1, \infty)}(k)=1\right\} .
$$

Proof. Let $\Delta_{1}(k)\left(\Delta_{2}(k)\right)$ denote the event that the departure of a cell of type 1 (type 2) occurs at time $k$. Note that these events are disjoint. Let $\Delta(k)$ denote $\Delta_{1}(k) \cup \Delta_{2}(k)$. Thus $\Delta(k)=\left\{d^{(1, \infty)}(k)=1\right\}$.

We observe that for $m \geqslant 1$ we have $\Delta_{1}(k+m) \subseteq \Delta(k)$. This is because if a departure of a cell of type 1 occurs at time $k+m$, there must have been cells of type 1 present in the system immediately after time $k$, so there must have been a departure of a cell of type 1 at time $k$ (recall the convention that the departures at time $k$ are determined after the arrival of cells at time $k$ ). 
We also observe that for any $m \geqslant 1, \Delta_{2}(k+m)$ and $\Delta(k)$ are independent. This is because the arrival process of sessions from time $k+1$ onwards is independent of the past and in particular is independent of whether there were departures at time $k$ or not (recall the convention that cells of type 2 have priority over cells of type 1).

With these two observations in mind, we have

$$
\begin{aligned}
P(\Delta(k+m), \Delta(k)) & =P\left(\Delta_{2}(k+m), \Delta(k)\right)+P\left(\Delta_{1}(k+m), \Delta(k)\right) \\
& =P\left(\Delta_{2}(k+m)\right) P(\Delta(k))+P\left(\Delta_{1}(k+m)\right)
\end{aligned}
$$

so that

$$
P_{1 \mid 1}^{m}=P(\Delta(k+m) \mid \Delta(k))=P\left(\Delta_{2}(k+m)\right)+\frac{P\left(\Delta_{1}(k+m)\right)}{P(\Delta(k))} .
$$

Further,

$$
P\left(\Delta(k+m), \Delta^{c}(k)\right)=P\left(\Delta_{2}(k+m), \Delta^{c}(k)\right)=P\left(\Delta_{2}(k+m)\right) P\left(\Delta^{c}(k)\right)
$$

so that

$$
P_{1 \mid 0}^{m}=P\left(\Delta(k+m) \mid \Delta^{c}(k)\right)=P\left(\Delta_{2}(k+m)\right) .
$$

From equations (8) and (9), we have

$$
P_{1 \mid 1}^{m}-P_{1 \mid 0}^{m}=\frac{P\left(\Delta_{1}(k+m)\right)}{P(\Delta(k))}=P\left(\Delta_{1}(k+m) \mid \Delta(k)\right),
$$

proving the proposition.

Let $Z_{k}^{*}$ denote the remaining number of cells just prior to time $k$, that it is already known will have to be handled by the system. Thus $Z_{k}^{*}$ consists of the cells that are in the cell buffer immediately before time $k$ as well as all the future cells of the sessions that are still active immediately before time $k$ that have not yet arrived in the system. Moreover, let $\phi_{k}$ be the total number of cells that are brought in by the sessions that are initiated at time $k$, i.e., the cells that they bring in at time $k$ and all future cell that they will bring in. Then the number of cells of type 1 that the system will have to handle is seen to be equal to

$$
N_{1}=\left(Z_{k}^{*}+\phi_{k}-1\right)^{+},
$$

where we take into account that in the time slot $[k, k+1)$ at most one cell of type 1 leaves the system. (In the preceding equation $x^{+}=\max \{0, x\}$.) The random variables $Z_{k}^{*}$ and $\phi_{k}$ are assumed to have their stationary distribution in the analysis that follows.

According to the discussion so far, in order to show that the departure process is long-range dependent it suffices to show that

$$
\sum_{m=1}^{\infty} P\left(\Delta_{1}(k+m)\right)=\infty .
$$


We have

$$
\begin{aligned}
\sum_{m=1}^{\infty} P\left(\Delta_{1}(k+m)\right) & =\sum_{m=1}^{\infty} \sum_{i=1}^{\infty} P\left(\Delta_{1}(k+m), N_{1}=i\right) \\
& =\sum_{i=1}^{\infty} \sum_{m=1}^{\infty} P\left(\Delta_{1}(k+m), N_{1}=i\right) \\
& =\sum_{i=1}^{\infty} P\left(N_{1}=i\right) \sum_{m=1}^{\infty} P\left(\Delta_{1}(k+m) \mid N_{1}=i\right) \\
& =\sum_{i=1}^{\infty} i P\left(N_{1}=i\right)=E\left[N_{1}\right],
\end{aligned}
$$

where we can justify the interchange in the order of summation in going from the first to the second line because the summands are nonnegative, and we can justify the next to last step because - due to the stability assumption - all cells of type 1 have to eventually leave the system. To see that note:

$$
\sum_{m=1}^{\infty} P\left(\Delta_{1}(k+m) \mid N_{1}=i\right)=E\left[\sum_{m=1}^{\infty} I\left(\Delta_{1}(k+m)\right) \mid N_{1}=i\right]=E[i]=i,
$$

where the second equation follows from the above argument.

Now from lemma 3.2 of [1] we have that

$$
P\left(Z_{k}^{*}>x\right) \sim \frac{\lambda}{(\alpha-1)(1-\lambda E \tau)} x^{1-\alpha} L(x),
$$

where $1<\alpha<2$ is the parameter of the regularly varying tail of the session duration and $L(\cdot)$ is a slowly varying function. From equations (11), (13) and (14), it follows immediately that equation (12) holds, showing that the stationary departure process of the leaky bucket system with infinite cell buffer in the case $C=1$ is indeed long-range dependent.

To establish the lower bound for the covariance of the departure process, we argue as follows: from (6) and (10) it suffices to consider $P\left(\Delta_{1}(k+m)\right)$. Let us denote by $N_{2}^{m}$ the number of cells of type 2 that arrive during the time $k+1, \ldots, k+m$. We also denote by $Q_{2}^{m}$ the number of cells of type 2 that are waiting in the queue for service at time $k+m$, including the arrivals at time $k+m$ and just prior to the service at that time. Recall that $N_{1}$ denotes the number of cells that belong to sessions which were initiated at or before time $k$ and which have not yet been served after service at time $k$, see equation (11). Now

$$
\begin{aligned}
P\left(\Delta_{1}(k+m)\right) & =\sum_{i=1}^{m} P\left(N_{2}^{m}=i-1, Q_{2}^{m}=0, N_{1}>m-i\right) \\
& =\sum_{i=1}^{m} P\left(N_{2}^{m}=i-1, Q_{2}^{m}=0\right) P\left(N_{1}>m-i\right)
\end{aligned}
$$




$$
\begin{aligned}
& \geqslant \sum_{i=1}^{m} P\left(N_{2}^{m}=i-1, Q_{2}^{m}=0\right) P\left(N_{1}>m\right) \\
& =P\left(N_{2}^{m}<m, Q_{2}^{m}=0\right) P\left(N_{1}>m\right),
\end{aligned}
$$

where the first step is because if a departure of type 1 occurs at time $k+m$ then the number of cells of type 2 arriving at times $k+1, \ldots, k+m$ is $m-1$ or less and, if this number is $i$, then at least $m-i$ cells of type 1 should exist, because there would have to have been a departure of a cell of type 1 at every one of the remaining $m-i$ times, and, further, no cells of type 2 could have been waiting for service at time $k+m$, while conversely, if $i \leqslant m-1$ cells of type 2 arrive, and there are at least $m-i$ cells of type 1 , and there are no cells of type 2 waiting for service at time $k+m$, then there must be a departure of type 1 at time $k+m$. The second step is by the independence of events associated with type 2 cells from the random variable $N_{1}$.

Now, as $m \rightarrow \infty$, the quantity $P\left(N_{2}^{m}<m, Q_{2}^{m}=0\right)$ converges to a nonzero constant, because the rate of arrivals of cells of type 2 is less than 1 and the arrival process is ergodic. Further, as a consequence of equation (14), $P\left(N_{1}>m\right)$ is asymptotically lower bounded by the right hand side of (14). The claimed lower bound (2), for an explicitly known constant $K_{1}$, follows.

For the upper bound we have the following:

$$
P\left(\Delta_{1}(k+m)\right) \leqslant P\left(N_{1}+N_{2}^{m} \geqslant m\right) .
$$

Indeed, if there is a departure of type 1 at time $k+m$, then there must be a departure at every one of the times $k+1, \ldots, k+m$, so that the total number of cells arriving at these times (i.e., $N_{2}^{m}$ ) plus the total number of cell of type 1 must be at least $m$. We now recall that $\phi_{k}$ denotes the total number of cells that are brought in by sessions initiated at time $k$ (see (11) and its explanation). We have the following relations:

$$
\begin{aligned}
P\left(N_{2}^{m}>m\right) & \leqslant P\left(\phi_{k+1}+\phi_{k+2}+\cdots+\phi_{k+m}>m\right) \\
& =P\left(\phi_{-(m+1)}+\phi_{-m}+\cdots+\phi_{-1}>m\right) \\
& \leqslant P\left(\widetilde{Z}_{0}>\eta m\right) .
\end{aligned}
$$

In the above, the inequality (17) is immediate, relation (18) holds because of stationarity, and the quantity $\widetilde{Z}_{0}$ gives the stationary number of cells just prior to time 0 , that it is known will have to be handled by the system when the service rate of the queue is $1-\eta$ with $\eta>0$ being such that $\lambda E \tau<1-2 \eta$. Now, from [1, lemma 3.2] we have

$$
P\left(\widetilde{Z}_{0}>x\right) \sim \frac{\lambda}{(\alpha-1)(1-\eta-\lambda E \tau)} x^{1-\alpha} L(x) .
$$

Using a well known result of regularly varying distributions regarding the sum of two independent regularly varying random variables (see, e.g., [6, p. 278]), the desired upper bound (3) follows, for a constant $K_{2}$ that can be explicitly estimated. 
It is interesting at this point to derive the asymptotic behaviour of the covariance sequence for the input process and compare it with the results obtained for the output process. The stationary version of the input process can be written as follows (see [10]):

$$
A(t)=\sum_{j=0}^{A(0)} I\left(\hat{\tau}_{j}>t\right)+A^{t}(1, t),
$$

where $A(0)$ is a Poisson random variable with parameter $\lambda E \tau, \hat{\tau}_{j}$ are i.i.d. random variables with distribution equal to the residual lifetime of the session duration $\tau$, and $A^{t}(1, t)$ is the number of cells at time $t$ due to sessions initiated between times 1 and $t$. It is obvious that $A^{t}(1, t)$ is independent of the variables $A(0)$ and $\hat{\tau}_{j}$, and it is also true that the random variables $A(0)$ and $\hat{\tau}_{j}$ are independent of each other. We have for the correlation of the input process

$$
\begin{aligned}
E[A(0) A(t)] & =E\left[b E\left[\sum_{j=0}^{b} I\left(\hat{\tau}_{j}>t\right) \mid A(0)=b\right]\right]+E[A(0)] E\left[A^{t}(1, t)\right] \\
& =E\left[A(0)^{2}\right] P(\hat{\tau}>t)+E[A(0)] E\left[A^{t}(1, t)\right] \\
& =\left(\lambda E \tau+(\lambda E \tau)^{2}\right) P(\hat{\tau}>t)+E[A(0)] E\left[A^{t}(1, t)\right] .
\end{aligned}
$$

Thus, for the covariance we have

$$
\begin{aligned}
E[ & A(0) A(t)]-E[A(0)] E[A(t)] \\
= & \left(\lambda E \tau+(\lambda E \tau)^{2}\right) P(\hat{\tau}>t)+E[A(0)] E\left[A^{t}(1, t)\right]-(\lambda E \tau)^{2} P(\hat{\tau}>t) \\
& \quad-E[A(0)] E\left[A^{t}(1, t)\right] \\
= & \lambda E \tau P(\hat{\tau}>t) \sim \frac{\lambda}{\alpha-1} t^{1-\alpha} L(t),
\end{aligned}
$$

where the last step regarding the asymptotic behaviour of the residual lifetime is a wellknown result (see, e.g., [4, proposition 1.5.10]). We have therefore found estimates for the decay of the autocorrelation of the departure process that are of the same order as those for the input process.

We will now consider the case of $C>1$. In keeping with the notation used above we denote by $\left\{d^{(C, \infty)}(k)\right\}$ the stationary departure process of the leaky bucket system in the case that the token buffer size is $C$ and the cell buffer size is infinite. In what follows we will refer to the leaky bucket system with token buffer size equal to $C$ (1) as the $C$-system (1-system). The key observation we need is the following lemma:

Lemma 2. It is possible to construct stationary versions of the $C$-system and the 1-system on the same sample space such that for every sample path of the arrival process the following inequalities hold:

$$
\begin{aligned}
& d^{(1, \infty)}(k)+d^{(1, \infty)}(k+1)+\cdots+d^{(1, \infty)}(k+m) \\
& \quad \leqslant d^{(C, \infty)}(k)+d^{(C, \infty)}(k+1)+\cdots+d^{(C, \infty)}(k+m)+(C-1),
\end{aligned}
$$




$$
\begin{gathered}
d^{(1, \infty)}(k)+d^{(1, \infty)}(k+1)+\cdots+d^{(1, \infty)}(k+m)+(C-1) \\
\geqslant d^{(C, \infty)}(k)+d^{(C, \infty)}(k+1)+\cdots+d^{(C, \infty)}(k+m),
\end{gathered}
$$

for any $k$ and any $m \geqslant 0$.

Proof. We will use the pathwise construction of the departure process of a leaky bucket system presented in [2]. Recall that $X^{(C, \infty)}(k),\left(Y^{(C, \infty)}(k)\right)$ denote the number of cells (tokens) in the cell (token) buffer at time $k$ in the system with token buffer size $C$. Obviously,

$$
0 \leqslant X^{(C, \infty)}(k)<\infty \quad \text { and } \quad 0 \leqslant Y^{(C, \infty)}(k) \leqslant C .
$$

We further note that $X^{(C, \infty)}(k) Y^{(C, \infty)}(k)=0$ for all $k$, so that the system can be described by the parameter

$$
Z^{(C, \infty)}(k)=X^{(C, \infty)}(k)-Y^{(C, \infty)}(k) .
$$

If $Z^{(C, \infty)}(k)>0$ there are $Z^{(C, \infty)}(k)$ cells and no tokens, if $-C \leqslant Z^{(C, \infty)}(k)<0$ there are $-Z^{(C, \infty)}(k)$ tokens and no cells, and if $Z^{(C, \infty)}(k)=0$ then there are neither cells nor tokens. Let $a(k)$ denote the number of cells arriving into the leaky bucket at time $k$. Then $Z^{(C, \infty)}(k)$ is given by the recursion

$$
Z^{(C, \infty)}(k+1)=Z^{(C, \infty)}(k)+a(k+1)-I\left\{Z^{(C, \infty)}(k) \geqslant-C+1\right\},
$$

where $I(A)$ denotes the indicator random variable of the event $A$. If we now define the quantity

$$
W(k)=Z^{(C, \infty)}(k)+C=X^{(C, \infty)}(k)-Y^{(C, \infty)}(k)+C,
$$

we get the following recursion for $W(k)$ from (26):

$$
W(k+1)=W(k)+a(k+1)-I\{W(k)>0\}=(W(k)-1)^{+}+a(k+1) .
$$

Note that $W(k) \geqslant 0, \forall k$. Relation (27) is nothing more than a special case of the familiar Lindley recursion from basic queueing theory [12] and from [9] we know that, since the stability condition $\lambda E \tau<1$ holds, there is a unique stationary and ergodic process that satisfies the recursion (27). Note that the distribution of $W$ does not depend on the choice of the token buffer size $C$. Nevertheless, the distributions of $Z^{(C, \infty)}=W-C, X^{(C, \infty)}=\max \{W-C, 0\}$ and $Y^{(C, \infty)}=\max \{C-W, 0\}$ do depend on $C$, as they should.

The stationary departure process of the leaky bucket system for any $C$ can be constructed from the stationary sample path of the $W$ process as shown in figure 3 . In order to determine the number of cells $d^{(C, \infty)}(k)$ that leave the $C$-system at time $k$ we consider the following cases in terms of the transition $W(k-1) \rightarrow W(k)$ :

(a) $W(k-1)=0$. In that case the number of departing cells is $d^{(C, \infty)}(k)=$ $\min \{W(k), C\}$. 


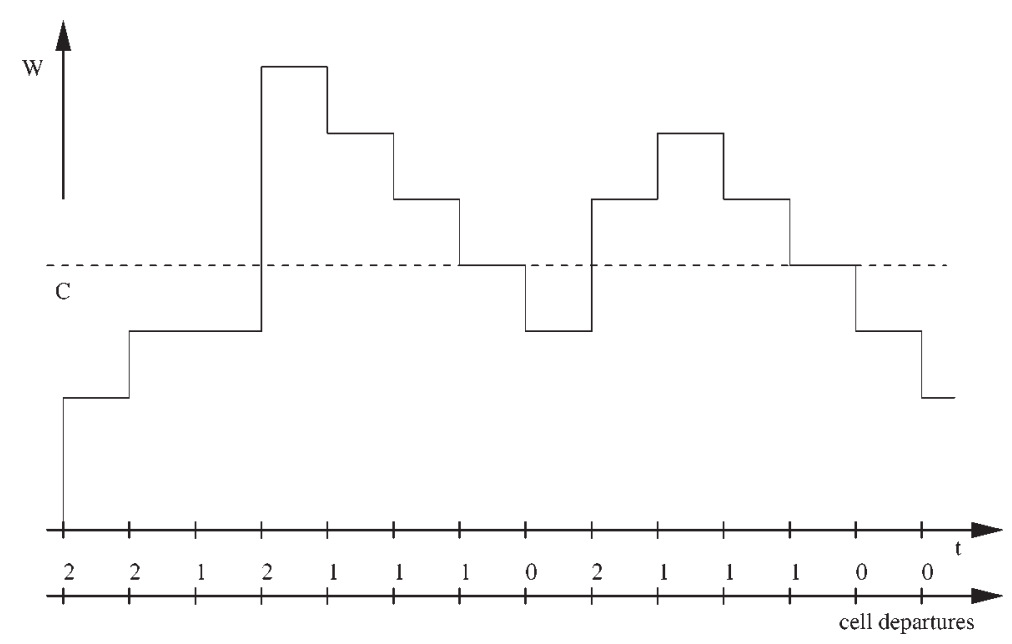

Figure 3. Construction of the departure process. The numbers on the axis labeled 'cell departures' give the number of cells leaving the system at each time instant for the specific realization of the process $W$.

(b) $0<W(k-1) \leqslant C$. In that case the number of departing cells is $d^{(C, \infty)}(k)=$ $\min \{W(k), C\}-W(k-1)+1$.

(c) $W(k-1)>C$. In that case there is exactly one departure at time $k$ due to the arriving token, i.e., $d^{(C, \infty)}(k)=1$.

The above considerations hold for any token buffer size and therefore also for $C=1$. This means that the departure processes for a whole range of systems, corresponding to different values of $C$, can be read off directly from the sample path of $W$. Note that for $W(k-1)>C$ we can only have one departure at any time instant.

Let

$$
d_{k, m}^{(C, \infty)}=d^{(C, \infty)}(k)+\cdots+d^{(C, \infty)}(k+m)
$$

be the number of departing cells from the $C$-system during the interval $[k, k+m]$, $m>0$. Also let

$$
\Delta d_{k, m}=d_{k, m}^{(1, \infty)}-d_{k, m}^{(C, \infty)}
$$

be the excess of the number of departing cells from the 1-system over the $C$-system over this interval. We are interested in an upper bound for the quantity $\Delta d_{k, m}$. Using the above results (a)-(c) we have the following cases:

- $W(k-1) \in\{0,1\}$ and $W(k+m) \in\{0,1\}$. Then $\Delta d_{k, m}=0$.

- $W(k-1) \in\{0,1\}$ and $W(k+m) \in\{2, \ldots, C\}$. Then $\Delta d_{k, m}=1-W(k+m)$.

- $W(k-1) \in\{0,1\}$ and $W(k+m)>C$. Then $\Delta d_{k, m}=1-C$.

- $W(k-1) \in\{2, \ldots, C\}$ and $W(k+m) \in\{0,1\}$. Then $\Delta d_{k, m}=W(k-1)-1$.

- $W(k-1) \in\{2, \ldots, C\}$ and $W(k+m) \in\{2, \ldots, C\}$. Then $\Delta d_{k, m}=W(k-1)-$ $W(k+m)$. 
- $W(k-1) \in\{2, \ldots, C\}$ and $W(k+m)>C$. Then $\Delta d_{k, m}=W(k-1)-C$.

- $W(k-1)>C$ and $W(k+m) \in\{0,1\}$. Then $\Delta d_{k, m}=C-1$.

- $W(k-1)>C$ and $W(k+m) \in\{2, \ldots, C\}$. Then $\Delta d_{k, m}=C-W(k+m)$.

- $W(k-1)>C$ and $W(k+m)>C$. Then $\Delta d_{k, m}=0$.

We see that the greatest value of $\Delta d_{k, m}$ is achieved for any interval $[k, k+m]$ with $W(k-1) \geqslant C$ and $W(k+m) \leqslant 1$ and for such an interval we get $\Delta d_{k, m}=C-1$. Moreover, the lowest value of $\Delta d_{k, m}$ is achieved for any interval $[k, k+m]$ with $W(k-1) \leqslant 1$ and $W(k+m) \geqslant C$ and for such an interval we get $\Delta d_{k, m}=-(C-1)$. Hence,

$$
-(C-1) \leqslant d_{k, m}^{(1, \infty)}-d_{k, m}^{(C, \infty)} \leqslant C-1,
$$

for any $k$ and for all $m>0$, and the proof is complete.

Now we can prove the following:

Theorem 1. The stationary departure process of the leaky bucket system is longrange dependent for any token buffer size $C$. Furthermore, if we denote by $\hat{d}_{k . m}^{(C, \infty)}$ the averaged process associated with the departure process in the case of the token buffer size being equal to $C$, i.e.,

$$
\hat{d}_{k . m}^{(C, \infty)}=\frac{d^{(C, \infty)}(k m-m+1)+\cdots+d^{(C, \infty)}(k m)}{m}
$$

and denote its variance by $v_{m}^{(C, \infty)}$, we have that there exist constants $K_{1}$ and $K_{2}$, such that

$$
v_{m}^{(C, \infty)} \gtrsim K_{1} m^{1-\alpha} L(m)
$$

and

$$
v_{m}^{(C, \infty)} \lesssim K_{2} m^{1-\alpha} L(m) .
$$

Explicit estimates can be given for the constants $K_{1}$ and $K_{2}$ in terms of the parameters of the problem.

Proof. We have shown the long-range dependence of the output process for $C=1$. To prove this in the case $C>1$ we proceed as follows: both sides of (24) are nonnegative, so we may square both sides and the inequality will still hold. If we also take expectations on both sides and subtract the quantity $E\left[d^{(1, \infty)}(k)\right]^{2}=E\left[d^{(C, \infty)}(k)\right]^{2}=(\lambda E \tau)^{2}$ from each expectation term on both sides we get the following relation:

$$
\begin{aligned}
& (m+1) r^{(1, \infty)}(0)+2 \sum_{i=1}^{m}(m-i+1) r^{(1, \infty)}(i) \\
& \leqslant(m+1) r^{(C, \infty)}(0)+2 \sum_{i=1}^{m}(m-i+1) r^{(C, \infty)}(i)+2(m+1) C \lambda E \tau+C^{2},
\end{aligned}
$$


where in the above we took into account that the output process is stationary. Let

$$
\Sigma_{0}^{(C, \infty)}(l) \triangleq \sum_{i=0}^{l}\left|r^{(C, \infty)}(i)\right| \quad \text { and } \quad \Sigma_{1}^{(1, \infty)}(l) \triangleq \sum_{i=1}^{l} r^{(1, \infty)}(i) .
$$

Also let $d \triangleq C \lambda E \tau$ and $g \triangleq 2 C \lambda E \tau+C^{2}$. Since $r^{(1, \infty)}(0), r^{(C, \infty)}(0) \geqslant 0$ we get from (31) after a few simple algebraic manipulations

$$
\sum_{l=1}^{m}\left(\Sigma_{1}^{(1, \infty)}(l)-d\right) \leqslant \sum_{l=0}^{m} \Sigma_{0}^{(C, \infty)}(l)+g .
$$

In order to show that $\left\{d^{(C, \infty)}(k)\right\}$ is long-range dependent, it suffices to show that for every $M>0$ there exists some integer $L$, such that

$$
\Sigma_{0}^{(C, \infty)}(l)>M, \quad \forall l \geqslant L .
$$

Note that since $\Sigma_{0}^{(C, \infty)}(l)$ is monotone increasing in $l$, then if (33) holds for some in$\operatorname{dex} L$, it will definitely hold for all indices $l \geqslant L$. To show (33) we may argue by contradiction. Suppose that for some $M_{0}$ there is no integer $L$, such that $\Sigma_{0}^{(C, \infty)}(L)>M_{0}$. But, since $\lim _{l \rightarrow \infty} \Sigma_{1}^{(1, \infty)}(l)=\infty$, this means that there exists some integer $K$, such that

$$
\Sigma_{1}^{(1, \infty)}(l)-d>M_{0}>\Sigma_{0}^{(C, \infty)}(l), \quad \forall l \geqslant K .
$$

This contradicts the inequality in (32) and therefore we must have that (33) holds or equivalently that $\left\{d^{(C, \infty)}(k)\right\}$ is long-range dependent.

We consider now the averaged departure process as defined in (28). After straightforward algebraic calculations, we obtain

$$
v_{m}^{(C, \infty)}=\frac{1}{m} r^{(C, \infty)}(0)+\frac{2}{m^{2}} \sum_{i=1}^{m-1}(m-i) r^{(C, \infty)}(i),
$$

see [5]. From (31) using (34) we can immediately get

$$
\frac{1}{m} r^{(1, \infty)}(0)+\frac{2}{m^{2}} \sum_{i=1}^{m-1}(m-i) r^{(1, \infty)}(i)-\frac{2 C}{m} \lambda E \tau-\frac{C^{2}}{m^{2}} \leqslant v_{m}^{(C, \infty)} .
$$

From lemma 1 we know that there exists $M_{1}$, such that, for $m \geqslant M_{1}$

$$
\begin{aligned}
\frac{1}{m} r^{(1, \infty)}(0)+\frac{2}{m^{2}} \sum_{i=1}^{m-1}(m-i) r^{(1, \infty)}(i)-\frac{2 C}{m} \lambda E \tau-\frac{C^{2}}{m^{2}} \\
=\frac{1}{m} r^{(1, \infty)}(0)+\frac{2}{m^{2}} \sum_{i=1}^{M_{1}-1}(m-i) r^{(1, \infty)}(i)-\frac{2 C}{m} \lambda E \tau-\frac{C^{2}}{m^{2}} \\
\quad+\frac{2}{m^{2}} \sum_{i=M_{1}}^{m-1}(m-i) r^{(1, \infty)}(i)
\end{aligned}
$$




$$
\begin{aligned}
& \geqslant \mathrm{o}\left(m^{1-\alpha} L(m)\right)+\frac{2}{m^{2}} \sum_{i=M_{1}}^{m-1} K_{1}(m-i) i^{1-\alpha} L(i) \\
& \geqslant \mathrm{o}\left(m^{1-\alpha} L(m)\right)+\frac{2}{m} \sum_{i=M_{1}}^{m-1} K_{1}\left(i-\frac{i}{m}\right) i^{1-\alpha} L(i) \\
& \geqslant K_{1}^{\prime} m^{1-\alpha} L(m) .
\end{aligned}
$$

This yields the desired lower bound (29).

For the upper bound we proceed in an analogous manner. First, we use (25) to obtain the following inequality in a way similar to the derivation of (31):

$$
\begin{aligned}
& (m+1) r^{(1, \infty)}(0)+2 \sum_{i=1}^{m}(m-i+1) r^{(1, \infty)}(i)+2(m+1) C \lambda E \tau+C^{2} \\
& \geqslant(m+1) r^{(C, \infty)}(0)+2 \sum_{i=1}^{m}(m-i+1) r^{(C, \infty)}(i)
\end{aligned}
$$

or, equivalently,

$$
\frac{1}{m} r^{(1, \infty)}(0)+\frac{2}{m^{2}} \sum_{i=1}^{m-1}(m-i) r^{(1, \infty)}(i)+\frac{2 C}{m} \lambda E \tau+\frac{C^{2}}{m^{2}} \geqslant v_{m}^{(C, \infty)} .
$$

Following the same steps as in the derivation of (36) through (39) and using the upper bound (3) instead of the lower bound relation, we readily obtain the desired upper bound (30). The proof of the theorem is complete.

It should be noted here that although we did not yet prove explicit bounds for the covariance sequence of the departure process in the case of the general token buffer size, the bounds on the variance of the averaged sequence provide strong indication that an asymptotic behaviour with the same power of decay should be expected of the covariance sequence as well. This is in agreement with the result given in [5], where this relation is established in the special case when the exact asymptotics of the variance of the averaged process are known.

\section{Finite cell buffer case}

We now consider the case where the cell buffer is finite, $0 \leqslant B<\infty$. We first examine the departure process in the case $C=1$ for any $B \geqslant 0$. In this case the leaky bucket system is equivalent to a single-server queue that is served at a constant rate equal to one cell per unit time. We prove the following:

Lemma 3. The departure process of the leaky bucket system in the case $C=1$ is long-range dependent for any cell buffer size $B$. Moreover, if we denote by $r^{(1, B)}(m)$ 
the covariance of the output process for lag $m$, then there exists constants $K_{1}$ and $K_{2}$, such that the following relations hold:

$$
r^{(1, B)}(m) \gtrsim K_{1} m^{1-\alpha} L(m)
$$

and

$$
r^{(1, B)}(m) \lesssim K_{2} m^{1-\alpha} L(m) .
$$

Explicit estimates for the constants $K_{1}$ and $K_{2}$ can be derived in terms of the parameters of the problem.

Proof. Let the stationary departure process be denoted by $\left\{d^{(1, B)}(k)\right\}$. Since $C=1$, we have $d^{(1, B)}(k) \in\{0,1\}, \forall k$. Let

$$
P\left(d^{(1, B)}(k)=1\right) \triangleq p_{\text {busy }}<1 \quad \text { and } \quad P\left(d^{(1, B)}(k)=0\right) \triangleq p_{\text {idle }}
$$

in stationarity. Then $E\left[d^{(1, B)}(k)\right]=p_{\text {busy }}$ in the stationary regime. The covariance of the departure process is

$$
\begin{aligned}
r^{(1, B)}(m)= & E\left[\left(d^{(1, B)}(k)-p_{\text {busy }}\right)\left(d^{(1, B)}(k+m)-p_{\text {busy }}\right)\right] \\
= & p_{\text {busy }}^{2} P\left(d^{(1, B)}(k+m)=0, d^{(1, B)}(k)=0\right) \\
& -p_{\text {busy }}\left(1-p_{\text {busy }}\right) P\left(d^{(1, B)}(k+m)=0, d^{(1, B)}(k)=1\right) \\
& -p_{\text {busy }}\left(1-p_{\text {busy }}\right) P\left(d^{(1, B)}(k+m)=1, d^{(1, B)}(k)=0\right) \\
& +\left(1-p_{\text {busy }}\right)^{2} P\left(d^{(1, B)}(k+m)=1, d^{(1, B)}(k)=1\right) .
\end{aligned}
$$

In this section we define $P_{i \mid j}^{m} \triangleq P\left(d^{(1, B)}(k+m)=i \mid d^{(1, B)}(k)=j\right.$ ) (note that this is different from the usage of $P_{i \mid j}^{m}$ in the preceding section). From the above relation, we get

$$
\begin{aligned}
r^{(1, B)}(m)= & p_{\text {busy }}^{2}\left(1-p_{\text {busy }}\right) P_{0 \mid 0}^{m}-p_{\text {busy }}^{2}\left(1-p_{\text {busy }}\right) P_{0 \mid 1}^{m} \\
& -p_{\text {busy }}\left(1-p_{\text {busy }}\right)^{2} P_{1 \mid 0}^{m}+p_{\text {busy }}\left(1-p_{\text {busy }}\right)^{2} P_{1 \mid 1}^{m} \\
= & p_{\text {busy }}\left(1-p_{\text {busy }}\right)\left(P_{0 \mid 0}^{m}-P_{0 \mid 1}^{m}\right) \\
= & p_{\text {busy }}\left(1-p_{\text {busy }}\right)\left(P_{1 \mid 1}^{m}-P_{1 \mid 0}^{m}\right) .
\end{aligned}
$$

In the above we used the fact that

$$
P_{0 \mid 0}^{m}+P_{1 \mid 0}^{m}=P_{0 \mid 1}^{m}+P_{1 \mid 1}^{m}=1 \text {. }
$$

From equation (45) one can see that $r^{(1, B)}(m)$ is positive, since $0<p_{\text {busy }}<1$ and $P_{1 \mid 1}^{m}>P_{1 \mid 0}^{m}$, which is proved below.

We will henceforth refer to cells belonging to sessions that have been initiated at or before time $k$ as cells of type 1, while cells belonging to sessions that arrived after time $k$ will be referred to as cells of type 2 . Assume that beginning at time 
$k+1$ priority is given to cells of type 2 over cells of type 1 ; this does not change the departure process in any way, since it only counts the total number of cells departing at any time. It is also important to note that whenever cells need to be rejected because the cell buffer if full, cells of type 1 are rejected in preference to cells of type 2 . Thus, from the point of view of cells of type 2 , it is as if cells of type 1 don't exist. If at some point there are no cells of type 2 in the system, then the service of any remaining cells of type 1 may be resumed.

As in the preceding section, let $\Delta_{1}(k)\left(\Delta_{2}(k)\right)$ denote the event that the departure of a cell of type 1 (type 2) occurs at time $k$. Note that these events are disjoint. Let $\Delta(k)$ denote $\Delta_{1}(k) \cup \Delta_{2}(k)$. Thus $\Delta(k)=\left\{d^{(1, \infty)}(k)=1\right\}$. As before, for $m \geqslant 1$ we have $\Delta_{1}(k+m) \subseteq \Delta(k)$. Further, for any $m \geqslant 1, \Delta_{2}(k+m)$ and $\Delta(k)$ are independent, because the arrival process of sessions from time $k+1$ onwards is independent of the past and the cell rejection rules ensure that point of view of type 2 cells it is as if the cell buffer were empty at time $k$ (note that no information is conveyed via the token buffer, because it has size 1). Thus we have

$$
\begin{aligned}
P(\Delta(k+m), \Delta(k)) & =P\left(\Delta_{2}(k+m), \Delta(k)\right)+P\left(\Delta_{1}(k+m), \Delta(k)\right) \\
& =P\left(\Delta_{2}(k+m)\right) P(\Delta(k))+P\left(\Delta_{1}(k+m)\right)
\end{aligned}
$$

so that

$$
P_{1 \mid 1}^{m}=P(\Delta(k+m) \mid \Delta(k))=P\left(\Delta_{2}(k+m)\right)+\frac{P\left(\Delta_{1}(k+m)\right)}{P(\Delta(k))} .
$$

Further,

$$
P\left(\Delta(k+m), \Delta^{c}(k)\right)=P\left(\Delta_{2}(k+m), \Delta^{c}(k)\right)=P\left(\Delta_{2}(k+m)\right) P\left(\Delta^{c}(k)\right)
$$

so that

$$
P_{1 \mid 0}^{m}=P\left(\Delta(k+m) \mid \Delta^{c}(k)\right)=P\left(\Delta_{2}(k+m)\right) .
$$

From equations (46) and (47), we have

$$
P_{1 \mid 1}^{m}-P_{1 \mid 0}^{m}=\frac{P\left(\Delta_{1}(k+m)\right)}{P(\Delta(k))}=P\left(\Delta_{1}(k+m) \mid \Delta(k)\right) .
$$

Thus, in order to show that the departure process is long-range dependent, it suffices to show that

$$
\sum_{m=1}^{\infty} P\left(\Delta_{1}(k+m)\right)=\infty .
$$

Let $S_{k}$ denote the set of sessions present in the system at time $k$ and let $X_{i}(k)$, $i \in S_{k}$, denote the number of cells belonging to session $i$ that arrive in the system after time $k$. We define

$$
X(k) \triangleq \max _{i \in S_{k}}\left\{X_{i}(k)\right\}
$$


Then we may write

$$
\begin{aligned}
P\left(\Delta_{1}(k+m)\right) & =\sum_{l=0}^{\infty} P\left(\Delta_{1}(k+m), X(k)=l\right) \\
& =\sum_{l=0}^{\infty} P(X(k)=l) P\left(\Delta_{1}(k+m) \mid X(k)=l\right) .
\end{aligned}
$$

We now use the following coupling argument: consider a second identical leaky bucket system with token buffer size $C=1$ and cell buffer size $B$, that is driven by an arrival process that is independent of and identically distributed to the arrival process of the original system up to and including time $k$. Starting at $k+1$ the part of the arrival process that consists of sessions that are generated at or after time $k+1$ is assumed to be pathwise the same for both systems. Of course, since the arrival processes in the two systems were independent for times prior to $k+1$, the part of the arrival process that consists of cells belonging to sessions which were generated before time $k+1$ will in general be different. In what follows we will refer to the cells which belong to sessions that were generated before or at time $k$ in the second system as cells of type 3 . Note that the cells of type 2 - as defined above - are common in both systems. It should also be pointed out that, by its definition, the second system is in stationarity at all times. Finally, we assume that after time $k$ we have the service discipline described above, i.e., cells of type 2 are given priority over cells of type 1 and 3 in the first (original) and second system respectively. Again, in the second system we require that when cells have to be rejected because the cell buffer is full, cells of type 3 are rejected in preference to cells of type 2 , so that, from the point of view of cells of type 2 , it is as if the cells of type 3 do not exist. Then, at any time instant, we may have only one of the following cases:

- There is no departure in any of the systems (case 0 ).

- A cell of type 1 departs in the original system and there is no departure in the second system (case 1).

- There is no departure in the original system and a cell of type 3 departs in the second system (case 2).

- A cell of type 1 departs in the original system and a cell of type 3 departs in the second system (case 3).

- A cell of type 2 departs in both systems (case 4).

We denote the events corresponding to the cases $0,1,2,3$ and 4 at time $j$ as $\Delta_{00}(j)$, $\Delta_{10}(j), \Delta_{03}(j), \Delta_{13}(j)$ and $\Delta_{22}(j)$, respectively.

With this notation, we may write, for any $l \geqslant 1$

$$
\begin{aligned}
& P\left(\Delta_{1}(k+m) \mid X(k)=l\right)=E\left[I\left(\Delta_{10}(k+m)\right)+I\left(\Delta_{13}(k+m)\right) \mid X(k)=l\right] \\
& \geqslant E\left[1-\left(I\left(\Delta_{03}(k+m)\right)+I\left(\Delta_{13}(k+m)\right)\right.\right. \\
& \left.\quad+I\left(\Delta_{22}(k+m)\right) \mid X(k)=l\right] \mathbf{1}(l \geqslant m),
\end{aligned}
$$


where in the above $I(A)$ is the indicator function of the event $A$. In writing the last equation we have observed that given $X(k)=l$, if $l \geqslant m$, there is always a departure in the original system at every time between $k+1$ and $k+m$. Since the second system is in stationarity despite the conditioning, we have that, if $l \geqslant m$,

$$
E\left[I\left(\Delta_{03}(k+m)\right)+I\left(\Delta_{13}(k+m)\right)+I\left(\Delta_{22}(k+m)\right) \mid X(k)=l\right]=p_{\text {busy }},
$$

where we used the fact that the union of the events in the summation is the event that at some time instant there is a departure in the second system. Thus we get, for any $l \geqslant 1$

$$
P\left(\Delta_{1}(k+m) \mid X(k)=l\right) \geqslant p_{\text {idle }} \mathbf{1}(l \geqslant m)
$$

so that

$$
P\left(\Delta_{1}(k+m)\right) \geqslant \sum_{l=0}^{\infty} P(X(k)=l) p_{\text {idle }} \mathbf{1}(l \geqslant m)=p_{\text {idle }} P(X(k) \geqslant m) .
$$

Now the quantity $X(k)$ is seen, from equation (21), to be the integer part of the maximum of a random number of independent random variables each having the distribution of the residual lifetime of the session duration $\tau$, where the number of such variables is Poisson with parameter $\lambda E \tau$. The tail probability $P(X(k) \geqslant m)$ is therefore asymptotically lower bounded by a constant times the tail probability $P(\hat{\tau} \geqslant m)$, where $\hat{\tau}$ has the distribution of the residual lifetime corresponding to $\tau$. From this the claimed lower bound (42) follows, using (23), as also the fact that

$$
\sum_{m=1}^{\infty} P\left(\Delta_{1}(k+m)\right) \geqslant p_{\text {idle }} \sum_{m=1}^{\infty} P(X(k) \geqslant m)=\infty,
$$

which establishes that the departure process of the leaky bucket is long-range dependent.

For the upper bound (43) we observe that

$$
P\left(\Delta_{1}(k+m)\right) \leqslant P(X(k)+B>m),
$$

because it is impossible to have a departure of type 1 at time $k+m$ unless there are cells of type 1 present. Appealing once again to the observation that $X(k)$ is maximum of a random number of independent random variables each having the distribution of the residual lifetime of the session duration $\tau$, where the number of such variables is Poisson, noting that the maximum is upper bounded by the sum, and appealing to the result [6, problem 31, p. 288], yields the desired upper bound. Explicit estimates for the constants $K_{1}$ and $K_{2}$ appearing in the equations (42) and (43) can be easily written down by going more carefully through the preceding argument. This concludes the proof of the lemma.

We will now consider the general case of $C>1$. We denote by $\left\{d^{(C, B)}(k)\right\}$ the stationary departure process of the leaky bucket system in the case that the token 
buffer size is $C$ and the cell buffer size is $B$. In what follows, we will call the corresponding system the $(C, B)$-system. The relevant range of the parameters is $C \geqslant 1$ and $0 \leqslant B<\infty$. We will first prove the following:

Lemma 4. For every $\left(C_{1}, B_{1}\right)$ and $\left(C_{2}, B_{2}\right)$ such that $C_{1}+B_{1}=B_{2}+C_{2}$ and $C_{1} \leqslant C_{2}$, it is possible to construct the $\left(C_{1}, B_{1}\right)$-system and the $\left(C_{2}, B_{2}\right)$-system on the same sample space such that, for every sample path of the arrival process the following inequalities hold:

$$
\begin{aligned}
& d^{\left(C_{1}, B_{1}\right)}(k)+d^{\left(C_{1}, B_{1}\right)}(k+1)+\cdots+d^{\left(C_{1}, B_{1}\right)}(k+m) \\
& \quad \leqslant d^{\left(C_{2}, B_{2}\right)}(k)+d^{\left(C_{2}, B_{2}\right)}(k+1)+\cdots+d^{\left(C_{2}, B_{2}\right)}(k+m)+\left(C_{2}-C_{1}\right), \\
& d^{\left(C_{1}, B_{1}\right)}(k)+d^{\left(C_{1}, B_{1}\right)}(k+1)+\cdots+d^{\left(C_{1}, B_{1}\right)}(k+m) \\
& \geqslant d^{\left(C_{2}, B_{2}\right)}(k)+d^{\left(C_{2}, B_{2}\right)}(k+1)+\cdots+d^{\left(C_{2}, B_{2}\right)}(k+m)-\left(C_{2}-C_{1}\right),
\end{aligned}
$$

for any $k$ and any $m \geqslant 0$.

Proof. We will again use the pathwise construction of the departure process of a leaky bucket system presented in [2]. Recall that $X^{(C, B)}(k),\left(Y^{(C, B)}(k)\right)$ denote the number of cells (tokens) in the cell (token) buffer at time $k$ in the $(C, B)$-system. Obviously, $0 \leqslant X^{(C, B)}(k) \leqslant B$ and $0 \leqslant Y^{(C, B)}(k) \leqslant C$. As discussed in lemma 2, the system can be described by the parameter $Z^{(C, B)}(k)=X^{(C, B)}(k)-Y^{(C, B)}(k)$. Let $a(k)$ denote the number of cells arriving into the leaky bucket at time $k$. Then $Z^{(C, B)}(k)$ is given by the recursion

$$
Z^{(C, B)}(k+1)=\min \left\{B, Z^{(C, B)}(k)+a(k+1)-I\left\{Z^{(C, B)}(k) \geqslant-C+1\right\}\right\},
$$

where $I(A)$ denotes the indicator random variable of the event $A$. If we now define the quantity $W^{(C, B)}(k)=Z^{(C, B)}(k)+C=X^{(C, B)}(k)-Y^{(C, B)}(k)+C$, we get the following recursion for $W^{(C, B)}(k)$ from (58):

$$
\begin{aligned}
W^{(C, B)}(k+1) & =\min \left\{C+B, W^{(C, B)}(k)+a(k+1)-I\left\{W^{(C, B)}(k)>0\right\}\right\} \\
& =\min \left\{C+B,\left(W^{(C, B)}(k)-1\right)^{+}+a(k+1)\right\},
\end{aligned}
$$

where in the above $(x)^{+}=\max \{x, 0\}$. Note that $0 \leqslant W^{(C, B)}(k) \leqslant C+B, \forall k$. It is important also to point out that the recursion for $W^{(C, B)}$ depends on the quantities $C$ and $B$ only through the sum $C+B$. Hence, if for two systems $C_{1}+B_{1}=C_{2}+B_{2}$, then $W^{\left(C_{1}, B_{1}\right)}$ and $W^{\left(C_{2}, B_{2}\right)}$ satisfy the same recursion. This means that if the $\left(C_{1}, B_{1}\right)$ system and the $\left(C_{2}, B_{2}\right)$-system are assumed to be driven by the same sample path, then the corresponding stationary $W$-processes are also pathwise the same.

The stationary departure process from the $(C, B)$-system can be constructed from a sample path of the $W^{(C, B)}$ process as shown in figure 4 . In order to determine the number of cells $d^{(C, B)}(k)$ that leave the $(C, B)$-system at time $k$ we consider the following cases: 


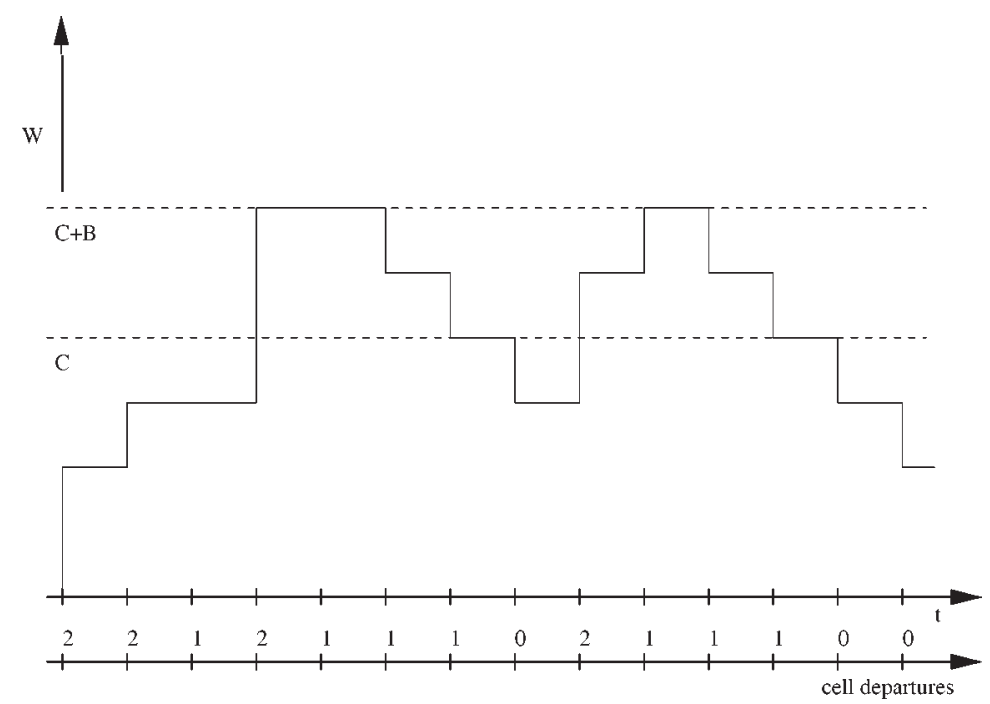

Figure 4. Construction of the departure process. The numbers on the axis labeled 'cell departures' give the number of cells leaving the system at each time instant for the specific realization of the process $W^{(C, B)}$.

(a) $W^{(C, B)}(k-1)=0$. In that case the number of departing cells is $d^{(C, B)}(k)=$ $\min \left\{W^{(C, B)}(k), C\right\}$.

(b) $0<W^{(C, B)}(k-1) \leqslant C$. In that case the number of departing cells is $d^{(C, B)}(k)=$ $\min \left\{W^{(C, B)}(k), C\right\}-W^{(C, B)}(k-1)+1$.

(c) $C<W^{(C, B)}(k-1) \leqslant C+B$. In that case there is exactly one departure at time $k$ due to the arriving token, i.e., $d^{(C, B)}(k)=1$.

Let

$$
d_{k, m}^{(C, B)}=d^{(C, B)}(k)+\cdots+d^{(C, B)}(k+m)
$$

be the stationary number of departing cells from the $(C, B)$-system during the interval $[k, k+m], m \geqslant 0$. Also let

$$
\Delta d_{k, m}=d_{k, m}^{\left(C_{1}, B_{1}\right)}-d_{k, m}^{\left(C_{2}, B_{2}\right)}
$$

be the excess in the number of departing cells from the $\left(C_{1}, B_{1}\right)$-system over the $\left(C_{2}, B_{2}\right)$-system over the same interval, where we recall that $C_{1}+B_{1}=C_{2}+B_{2}$. We are interested in upper and lower bounds for the quantity $\Delta d_{k, m}$. Recall that we have assumed that $C_{1} \leqslant C_{2}$. Using the above results (a)-(c) we have the following cases, where we take into account that the $W$-processes of the two systems are pathwise the same, as discussed above, and therefore are represented simply by $W(\cdot)$ :

- $W(k-1) \leqslant C_{1}$ and $W(k+m) \leqslant C_{1}$. Then $\Delta d_{k, m}=0$.

- $W(k-1) \leqslant C_{1}$ and $C_{1}+1 \leqslant W(k+m) \leqslant C_{2}$. Then $\Delta d_{k, m}=C_{1}-W(k+m)$.

- $W(k-1) \leqslant C_{1}$ and $C_{2}+1 \leqslant W(k+m) \leqslant C_{1}+B_{1}$. Then $\Delta d_{k, m}=C_{1}-C_{2}$. 
- $C_{1}+1 \leqslant W(k-1) \leqslant C_{2}$ and $W(k+m) \leqslant C_{1}$. Then $\Delta d_{k, m}=W(k-1)-C_{1}$.

- $C_{1}+1 \leqslant W(k-1) \leqslant C_{2}$ and $C_{1}+1 \leqslant W(k+m) \leqslant C_{2}$. Then $\Delta d_{k, m}=$ $W(k-1)-W(k+m)$.

- $C_{1}+1 \leqslant W(k-1) \leqslant C_{2}$ and $C_{2}+1 \leqslant W(k+m) \leqslant C_{1}+B_{1}$. Then $\Delta d_{k, m}=$ $W(k-1)-C_{2}$.

- $C_{2}+1 \leqslant W(k-1) \leqslant C_{1}+B_{1}$ and $W(k+m) \leqslant C_{1}$. Then $\Delta d_{k, m}=C_{2}-C_{1}$.

- $C_{2}+1 \leqslant W(k-1) \leqslant C_{1}+B_{1}$ and $C_{1}+1 \leqslant W(k+m) \leqslant C_{2}$. Then $\Delta d_{k, m}=$ $C_{2}-W(k+m)$.

- $C_{2}+1 \leqslant W(k-1) \leqslant C_{1}+B_{1}$ and $C_{2}+1 \leqslant W(k+m) \leqslant C_{1}+B_{1}$. Then $\Delta d_{k, m}=0$.

We see that the greatest value of $\Delta d_{k, m}$ is achieved for any interval $[k, k+m]$ with $C_{2} \leqslant W(k-1) \leqslant C_{1}+B_{1}$ and $W(k+m) \leqslant C_{1}$ and for these intervals we get $\Delta d_{k, m}=C_{2}-C_{1}$. Similarly, the smallest value is achieved for any interval $[k, k+m]$ with $W(k-1) \leqslant C_{1}$ and $C_{2} \leqslant W(k+m) \leqslant C_{1}+B_{1}$ and for these intervals we get $\Delta d_{k, m}=-\left(C_{2}-C_{1}\right)$. Hence,

$$
-\left(C_{2}-C_{1}\right) \leqslant d_{k, m}^{\left(C_{1}, B_{1}\right)}-d_{k, m}^{\left(C_{2}, B_{2}\right)} \leqslant C_{2}-C_{1}, \quad \forall m>0, k,
$$

if $C_{1}+B_{1}=C_{2}+B_{2}$ with $C_{1} \leqslant C_{2}$ and the proof is complete.

It should be noted here that due to the above lemma and the fact that the departure process for any $C$ and $B$ is stationary and ergodic, the expected values of the departure processes when the conditions $C_{1}+B_{1}=C_{2}+B_{2}, C_{1} \leqslant C_{2}$ hold are the same, since

$$
\begin{aligned}
\lim _{m \rightarrow \infty} \frac{d_{k, m}^{\left(C_{2}, B_{2}\right)}-\left(C_{2}-C_{1}\right)}{m} & \leqslant E\left[d^{\left(C_{1}, B_{1}\right)}\right]=\lim _{m \rightarrow \infty} \frac{d_{k, m}^{\left(C_{1}, B_{1}\right)}}{m} \\
& \leqslant \lim _{m \rightarrow \infty} \frac{d_{k, m}^{\left(C_{2}, B_{2}\right)}+\left(C_{2}-C_{1}\right)}{m}
\end{aligned}
$$

and the first and third limits are the same and equal to

$$
E\left[d^{\left(C_{2}, B_{2}\right)}\right]=\lim _{m \rightarrow \infty} \frac{d_{k, m}^{\left(C_{2}, B_{2}\right)}}{m} .
$$

Now we can prove the following:

Theorem 2. The departure process of the leaky bucket system is long-range dependent for any token buffer size $C \geqslant 1$ and any finite cell buffer size $0 \leqslant B<\infty$. Furthermore, if we denote by $\hat{d}_{k, m}^{(C, B)}$ the averaged process associated with the departure process in the case of the token buffer size being equal to $C$, i.e.,

$$
\hat{d}_{k . m}^{(C, B)}=\frac{d^{(C, B)}(k m-m+1)+\cdots+d^{(C, B)}(k m)}{m}
$$


and denote its variance by $v_{m}^{(C, B)}$, we have that there exist constants $K_{1}$ and $K_{2}$, such that the following relations hold: for $m>M_{1}$

$$
v_{m}^{(C, B)} \gtrsim K_{1} m^{1-\alpha} L(m)
$$

and

$$
v_{m}^{(C, B)} \lesssim K_{2} m^{1-\alpha} L(m) .
$$

The constants $K_{1}$ and $K_{2}$ can be explicitly estimated in terms of the parameters of the problem.

Proof. We have shown the result for $C=1$ and for any value of $B$. To prove the result in the case $C>1$ we proceed as follows: both sides of (56) are nonnegative, so we may square both sides and the inequality will still hold. We also assume that $C_{1}=1$. If we take expectations on both sides and subtract the quantity

$$
E^{2}=E\left[d^{\left(1, B_{1}\right)}(k)\right]^{2}=E\left[d^{\left(C_{2}, B_{2}\right)}(k)\right]^{2}
$$

from each expectation term on both sides we get the following relation:

$$
\begin{aligned}
& (m+1) r^{\left(1, B_{1}\right)}(0)+2 \sum_{i=1}^{m}(m-i+1) r^{\left(1, B_{1}\right)}(i) \\
& \leqslant(m+1) r^{\left(C_{2}, B_{2}\right)}(0)+2 \sum_{i=1}^{m}(m-i+1) r^{\left(C_{2}, B_{2}\right)}(i)+2(m+1) C_{2} E+C_{2}^{2},
\end{aligned}
$$

where in the above we took into account that the output process is stationary. Let

$$
\Sigma_{0}^{\left(C_{2}, B_{2}\right)}(l) \triangleq \sum_{i=0}^{l}\left|r^{\left(C_{2}, B_{2}\right)}(i)\right| \quad \text { and } \quad \Sigma_{1}^{\left(1, B_{1}\right)}(l) \triangleq \sum_{i=1}^{l} r^{\left(1, B_{1}\right)}(i) .
$$

Also let $d \triangleq C_{2} E$ and $g \triangleq 2 C_{2} E+C_{2}^{2}$. Since $r^{\left(1, B_{1}\right)}(0), r^{\left(C_{2}, B_{2}\right)}(0) \geqslant 0$ we get from (63) after a few simple algebraic manipulations

$$
\sum_{l=1}^{m}\left(\Sigma_{1}^{\left(1, B_{1}\right)}(l)-d\right) \leqslant \sum_{l=0}^{m} \Sigma_{0}^{\left(C_{2}, B_{2}\right)}(l)+g .
$$

In order to show that $\left\{d^{\left(C_{2}, B_{2}\right)}(k)\right\}$ is long-range dependent, it suffices to show that for every $M>0$ there exists some integer $L$, such that

$$
\Sigma_{0}^{\left(C_{2}, B_{2}\right)}(l)>M, \quad \forall l \geqslant L .
$$

Note that since $\Sigma_{0}^{\left(C_{2}, B_{2}\right)}(l)$ is monotone increasing in $l$, then if (65) holds for some index $L$, it will definitely hold for all indices $l \geqslant L$. To show (65) we may argue by contradiction. Suppose that for some $M_{0}$ there is no integer $L$, such that $\Sigma_{0}^{\left(C_{2}, B_{2}\right)}(L)>M_{0}$. 
But, since $\lim _{l \rightarrow \infty} \Sigma_{1}^{\left(1, B_{1}\right)}(l)=\infty$, this means that there exists some integer $K$, such that

$$
\Sigma_{1}^{\left(1, B_{1}\right)}(l)-d>M_{0}>\Sigma_{0}^{\left(C_{2}, B_{2}\right)}(l), \quad \forall l \geqslant K .
$$

This contradicts the inequality in (64) and therefore we must have that (65) holds or equivalently that $\left\{d^{(C, B)}(k)\right\}$ is long-range dependent for any values of $C$ and $B$.

We consider now the averaged departure process as defined in (60). After straightforward algebraic calculations, we obtain

$$
v_{m}^{(C, B)}=\frac{1}{m} r^{(C, B)}(0)+\frac{2}{m^{2}} \sum_{i=1}^{m-1}(m-i) r^{(C, B)}(i),
$$

see [5]. From (63) using (66) we can immediately get

$$
\frac{1}{m} r^{(1, B)}(0)+\frac{2}{m^{2}} \sum_{i=1}^{m-1}(m-i) r^{(1, B)}(i)-\frac{2 C}{m} \lambda E \tau-\frac{C^{2}}{m^{2}} \leqslant v_{m}^{(C, B)} .
$$

We may now proceed as was done in the cae $B=\infty$ to get the desired lower bound (61).

For the upper bound we proceed in an analogous manner. First, we use (57) to obtain the following inequality in a way similar to the derivation of (63):

$$
\begin{aligned}
& (m+1) r^{(1, B)}(0)+2 \sum_{i=1}^{m}(m-i+1) r^{(1, B)}(i)+2(m+1) C \lambda E \tau+C^{2} \\
& \geqslant(m+1) r^{(C, B)}(0)+2 \sum_{i=1}^{m}(m-i+1) r^{(C, B)}(i)
\end{aligned}
$$

or, equivalently,

$$
\frac{1}{m} r^{(1, B)}(0)+\frac{2}{m^{2}} \sum_{i=1}^{m-1}(m-i) r^{(1, B)}(i)+\frac{2 C}{m} \lambda E \tau+\frac{C^{2}}{m^{2}} \geqslant v_{m}^{(C, B)} .
$$

We may now proceed as was done in the cae $B=\infty$ to get the desired upper bound (62). The proof of the theorem is complete.

Again, we have found estimates for the variance of the averaged process associated to the departure process, which suggests that the corresponding bounds for the correlation function of the departure process should hold. However we have not yet established such bounds.

\section{Concluding remarks}

We have studied the departure process of a leaky bucket system in an ATM network fed by a class of proposed models for long-range dependent input traffic. We 
established the fact that the departure process is long-range dependent for any token buffer size and any - finite or infinite - cell buffer size. Moreover, we established bounds for the asymptotic behaviour of the covariance of the output process in the above cases. This result provides additional evidence supporting the importance of the empirical observations that there is long-range dependence in the offered traffic that broadband networks are expected to carry. Indeed, what it demonstrates is that this long-range dependence cannot be removed by the kinds of flow control schemes that are currently being envisioned for these networks.

\section{References}

[1] V. Anantharam, On the sojourn time of sessions at an ATM buffer with long-range dependent input traffic, in: Proc. of the 34rd IEEE Conf. on Decision and Control, Vol. 1, New Orleans, LA (December 13-15, 1995) pp. 859-864.

[2] V. Anantharam and T. Konstantopoulos, Burst reduction properties of the leaky bucket flow control scheme in ATM networks, IEEE Trans. Commun. 42(12) (1994) 3085-3089.

[3] J. Beran, R. Sherman, M.S. Taqqu and W. Willinger, Long-range dependence in variable-bit-rate video traffic, IEEE Trans. Commun. 43(2/3/4) (1995) 1566-1579.

[4] N.H. Bingham, C.M. Goldie and J.L. Teugels, Regular Variation (Cambridge University Press, New York, 1987).

[5] D.R. Cox, Long-range dependence: A review, in: Statistics: An Appraisal, eds. H.A. David and H.T. David (Iowa State University Press, 1984) pp. 55-74.

[6] W. Feller, An Introduction to Probability Theory and its Applications, Vol. 2 (Wiley, New York, 1971).

[7] W.E. Leland, M.S. Taqqu, W. Willinger and D.V. Wilson, On the self-similar nature of Ethernet traffic (extended version), IEEE/ACM Trans. Networking 2(1) (1994) 1-15.

[8] N. Likhanov, B. Tsybakov and N.D. Georganas, Analysis of an ATM buffer with self-similar ("fractal") input traffic, in: Proc. of the 14th Annual IEEE Infocom (1995) pp. 985-992.

[9] R.M. Loynes, The stability of queues with non-independent interarrival and service times, Proceedings of the Cambridge Philosophical Society 58 (1962) 497-520.

[10] M. Parulekar and A.M. Makowski, Tail probabilities for $M / G / \infty$ input processes (I): Preliminary asymptotics, Preprint (1997).

[11] V. Paxson and S. Floyd, Wide area traffic: The failure of Poisson modelling, IEEE/ACM Transactions on Networking 3(3) (1995) 226-244.

[12] J. Walrand, An Introduction to Queueing Networks (Prentice-Hall, Englewood Cliffs, NJ, 1988).

[13] D. Williams, Probability with Martingales (Cambridge University Press, New York, 1991). 